REVISTA

\title{
EFICIÊNCIA DE IRRIGAÇÃO EM CAFEEIROS CONILON NA REGIÃO CENTRO SERRANA DO ESPÍRITO SANTO
}

\author{
IRRIGATION EFFICIENCY IN CONILON COFFEE CROP IN THE \\ MONTAINEUS CENTRAL REGION OF ESPIRITO SANTO STATE
}

\author{
${ }^{1 *}$ Gustavo Haddad Souza Vieira. \\ ${ }^{1}$ Demétrius Pinto Nascimento. \\ ${ }^{1}$ Paola Alfonsa Vieira Lo Monaco. \\ ${ }^{1}$ Ismail Ramalho Hadadde. \\ ${ }^{1}$ Thiago Lopes Rosado. \\ ${ }^{1}$ Alberto Chambela Neto.
}

${ }^{1}$ Instituto Federal do Espírito Santo, Ifes Campus Santa Teresa, Santa Teresa-ES. E-mail: ghsv@ifes.edu.br.

*Autor de correspondência

Artigo submetido em 30/09/2020, aceito em 28/11/2020 e publicado em 23/11/2020.

\begin{abstract}
Resumo: O café representa a principal fonte de renda dos produtores rurais de São Roque do CanaãES, porém o município apresenta restrição hídrica ao cafeeiro devido à baixa e irregular distribuição de chuvas, o que implica na adoção da irrigação para suprir as exigências hídricas do cafeeiro, principalmente do tipo localizada por gotejamento. Porém, identifica-se a ausência de limpeza, manutenção e avaliações nestes sistemas de irrigação, o que reduz sua eficiência e uniformidade de aplicação de água. Diante do exposto, objetivou-se com a realização deste trabalho, avaliar o desempenho de sistemas de irrigação localizada por gotejamento no município de São Roque do Canaã, visando a determinação da uniformidade e da eficiência de aplicação de água. Foram avaliados sistemas de irrigação em dez propriedades no município. Com base nos resultados obtidos, foram determinados e classificados o Coeficiente de Uniformidade de Christiansen (CUC), o Coeficiente de Uniformidade de Distribuição (CUD), o Coeficiente de Uniformidade Estatística (Us) e a Eficiência de aplicação (Ea). Os sistemas de irrigação localizada por gotejamento apresentaram baixa uniformidade de aplicação de água, sendo necessário que algumas práticas de manutenção preventiva e corretiva sejam adotadas para que os sistemas operem com maior uniformidade e, em consequência, com maior eficiência no uso da água.
\end{abstract}

Palavras-chave: gotejamento; uniformidade; Coffea canephora; avaliação.

Abstract: Coffee represents the main source of income for rural producers in São Roque do Canaã, ES, Brazil. However, the municipality has severe water restrictions to the coffee crop due to the low and irregular distribution of rainfall, what implies the adoption of irrigation to supply the water requirements of the coffee crop, mainly the drip irrigation. However, the absence of cleaning, maintenance and assessment in these irrigation systems is identified, which reduces the efficiency and uniformity of water application. In view of the above, the objective of this study was to evaluate the performance of drip irrigation systems, in order to determine the uniformity and efficiency of water application. Irrigation systems were evaluated in ten small farms. Based on the results obtained, the Christiansen's Uniformity Coefficient (CUC), the Distribution Uniformity Coefficient (CUD), the 
Statistical Uniformity Coefficient (Us) and the Application Efficiency (Ea) were determined and classified. The drip irrigation systems showed low uniformity of water application, being necessary that some preventive and corrective maintenance practices be adopted so that the systems operate with higher uniformity, and consequently, with greater water use efficiency.

Keywords: drip irrigation; uniformity; Coffea canephora; assessment.

\section{INTRODUÇÃO}

O Espírito Santo é o maior produtor nacional de café Conilon (Coffea canephora), que está presente em 65 dos 78 municípios, sendo os maiores produtores Jaguaré, Vila Valério, Sooretama, Rio Bananal e São Gabriel da Palha (DARDENGO, 2012).

O café representa a principal fonte de renda dos produtores rurais do município de São Roque do Canaã, possuindo uma área plantada de 5.000 ha. No entanto, seu potencial produtivo ainda não é explorado em sua plenitude. A baixa produtividade das lavouras do município é causada pelo baixo potencial produtivo das mudas implantadas, correções e adubações do solo insuficientes e até inexistentes, além de inadequada distribuição de chuvas.

Segundo o CETCAF (2020) toda a área do município encontra-se classificada como área com altitudes inferiores a 500 metros e com restrição hídrica ao cafeeiro. Visando o suprimento da sua demanda hídrica, os produtores rurais instalaram sistemas de irrigação em suas lavouras. Em virtude do que é divulgado sobre sua maior eficiência, o sistema de irrigação localizada por gotejamento é o mais adotado na região.

Porém, a grande maioria destes sistemas conta com dois fatores limitantes ao seu funcionamento: o primeiro é a ausência de projeto técnico elaborado por profissional treinado e capacitado, considerando as características físicohídricas do solo em que a cultura está implantada; o outro fator que reduz o potencial destes sistemas é a ausência de manutenção preventiva periódica do equipamento de irrigação.
O sistema de irrigação localizada por gotejamento é conhecido por apresentar alto potencial de eficiência na aplicação de água e fertilizante. No entanto, tal aplicação deve ser associada a um projeto de irrigação bem dimensionado, que considera, entre outros fatores, o movimento de água no solo (BERNARDO et. al, 2019).

Este sistema apresenta vantagens em relação a outros sistemas, como potencial de eficiência de uso de água, facilitação de tratos culturais, maior eficiência no controle fitossanitário, visto que irriga menores áreas ocupadas por plantas invasoras e não molha a parte aérea dos vegetais, dentre outras. Como desvantagens, possui sensibilidade ao entupimento dos emissores, alto custo inicial, exigência em manutenção com maior frequência e complicações de uso em águas com altos teores de ferro.

A irrigação excessiva, o desperdício da água e o comprometimento na produção da lavoura contribuem para um maior impacto ambiental sobre o solo e a qualidade dos mananciais que recebem o excesso de água aplicada, carreando consigo parte dos insumos aplicados nos tratos culturais, como fertilizantes e agrotóxicos (RODRIGUES et al., 2013).

Neste sentido, Vieira et al. (2004) relatam que a uniformidade de distribuição de água pelo sistema de irrigação é um dos aspectos mais importantes a serem observados no manejo da irrigação. Bernardo et al. (2019) afirmam que o quanto maior a uniformidade de distribuição de água, menor será a lâmina de irrigação necessária para alcançar a produtividade máxima. O conhecimento desta uniformidade implica na medição das vazões dos gotejadores ao longo das linhas 
laterais (VIEIRA; MANTOVANI; CORDEIRO, 2003).

A desuniformidade de aplicação de água faz com que o sistema aplique água em uma fração da área com excesso, onde uma parte é perdida por percolação e transporta consigo parte dos nutrientes presentes na zona de aplicação e outra fração, em déficit, em que a água infiltrada fica armazenada na zona radicular, porém em quantidade inferior à necessidade da planta (ZOCOLER, 2015). Faria et al. (2004) destacam que os fatores que influenciam na uniformidade de aplicação de água pelo sistema de irrigação localizada por gotejamento seguem a ordem: obstruções dos emissores, coeficiente de variação de fabricação, expoente de descarga do emissor, sensibilidade do emissor à temperatura e variações de pressão, dentre outros. Esta obstrução é causada por material orgânico em suspensão, por deposição química e por partículas minerais, características hidráulicas, topografia do terreno, pressão de operação, tamanho dos tubos, espaçamento entre emissores, variabilidade de vazão dos emissores e filtragem da água não adequada (CARVALHO et al., 2006).

Assim, este trabalho teve como objetivo realizar um diagnóstico de sistemas de irrigação localizada por gotejamento instalados em lavouras de cafeeiro Conilon no município de São Roque do Canaã, ES.

\section{MATERIAIS E MÉTODOS}

\begin{tabular}{ccr} 
O & presente & \multicolumn{2}{c}{ trabalho foi } \\
desenvolvido & em dez propriedades
\end{tabular} produtoras de café Conilon, que utilizam o sistema de irrigação localizada por gotejamento, no município de São Roque do Canaã (Figura 1).

Figura 1: Localização dos sistemas de irrigação localizada por gotejamento avaliados no município de São Roque do Canaã.

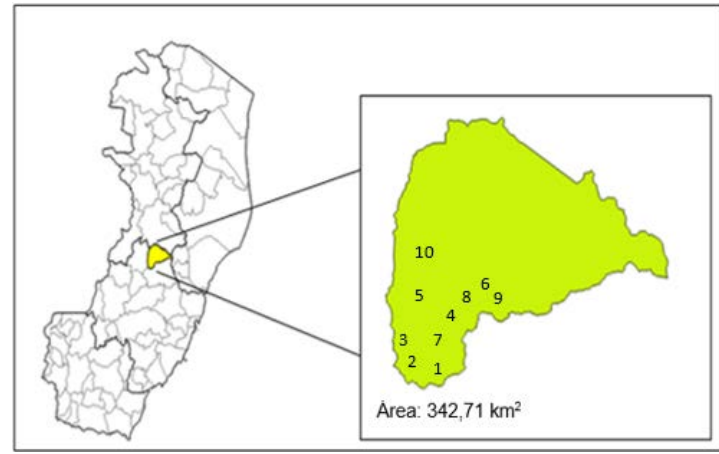

Fonte: Adaptado de IBGE (2014)

A seleção das propriedades rurais e sua localização foram realizadas baseandose em levantamentos realizados junto INCAPER. Como critério, foram selecionados os sistemas de irrigação localizada por gotejamento, com linhas laterais em nível. A Tabela 1 apresenta as características dos sistemas avaliados, como área total do sistema de irrigação, área do setor avaliado, tempo de uso dos sistemas e idade do cafeeiro.

Tabela 1: Caracterização dos sistemas avaliados, com área total e do setor, tempo de uso do sistema e idade da lavoura

\begin{tabular}{|c|c|c|c|c|}
\hline \multirow[t]{2}{*}{ Sistema } & $\begin{array}{c}\text { Área } \\
\text { total } \\
\text { irrigada }\end{array}$ & $\begin{array}{c}\text { Área do } \\
\text { setor } \\
\text { avaliado }\end{array}$ & \multicolumn{2}{|c|}{ Idade (anos) } \\
\hline & \multicolumn{2}{|c|}{-------ha------- } & Sistema & Lavoura \\
\hline 1 & 2,0 & 2,0 & 1,0 & 2,0 \\
\hline 2 & 7,6 & 1,2 & 2,0 & 2,0 \\
\hline 3 & 12,0 & 1,4 & 1,5 & 1,5 \\
\hline 4 & 9,2 & 0,9 & 1,8 & 1,8 \\
\hline 5 & 1,6 & 0,4 & 3,0 & 3,0 \\
\hline 6 & 3,0 & 0,8 & 2,0 & 2,0 \\
\hline 7 & 4,3 & 1,2 & 5,0 & 5,0 \\
\hline 8 & 5,8 & 1,4 & 3,5 & 3,5 \\
\hline 9 & 6,0 & 1,5 & 3,0 & 6,0 \\
\hline 10 & 5,5 & 1,0 & 3,0 & 7,0 \\
\hline
\end{tabular}

Fonte: Elaborado pelos autores

Foram avaliados tanto os sistemas de irrigação com projeto técnico e outros com ausência de projeto, possibilitando a comparação entre eles. Dentre os setores do sistema de irrigação de cada propriedade, a escolha do setor avaliado foi realizada, aleatoriamente, através de sorteio.

A caracterização hidráulica dos sistemas de irrigação contou com a 
determinação da pressão e da vazão dos emissores e do sistema em geral. A determinação da uniformidade de distribuição de água foi realizada seguindose o método proposto por Keller e Karmeli (1975), alterado por Denículi et al. (1980) e apresentado por Mantovani et al. (2009). A pressão de serviço do sistema foi aferida com utilização de manômetro metálico graduado de 0 a $100 \mathrm{kPa}$, acoplado à bomba, no início (quando as linhas de derivação não estavam enterradas) e no final de cada linha lateral avaliada.

A determinação de área molhada por cada emissor foi calculada de acordo com a metodologia de Merriam e Keller, adaptada por Bonomo (1999). A determinação ocorreu após conclusão do tempo total de irrigação, com avaliação de três plantas, em sequência, nas quatro linhas laterais em que foram medidas as vazões. A porcentagem de área molhada (PAM) e a porcentagem de área sombreada (PAS) foram determinadas de acordo com Bernardo et al. (2019). Posteriormente às coletas de dados em campo, foram realizados os cálculos para diagnóstico dos sistemas de irrigação avaliados.

A lâmina aplicada (Lapl) pelo emissor foi quantificada, considerando-se o tempo total de irrigação adotado pelo produtor, utilizando a Equação 1 (BONOMO, 1999). Este parâmetro indica o quanto de água é emitido pelo gotejador, possibilitando sua comparação de operação com o que é descrito pelo fabricante do equipamento.

$$
\mathrm{L}_{\mathrm{apl}}=\frac{Q T}{A}
$$

Em que:

Lapl - lâmina aplicada média (mm);

$\mathrm{Q}$ - vazão média do emissor $\left(\mathrm{L} \mathrm{h}^{-1}\right)$;

$\mathrm{T}$ - tempo (h);

A - área representada pelo emissor $\left(\mathrm{m}^{2}\right)$.
Com os dados de vazão, o Coeficiente de Uniformidade de Christiansen (CUC) foi determinado para expressar a uniformidade de aplicação de água em sistemas de irrigação localizada, conforme apresentado na Equação 2.

$$
\mathrm{CUC}=100\left(1-\frac{\sum_{i=1}^{n}\left|q_{i}-\mathrm{q}_{\mathrm{m}}\right|}{\mathrm{n} \mathrm{\textrm {q } _ { \mathrm { m } }}}\right)
$$

Em que:

CUC - coeficiente de uniformidade de Christiansen (\%);

$\mathrm{q}_{\mathrm{i}}$ - vazão de cada gotejador $\left(\mathrm{L} \mathrm{h}^{-1}\right)$;

$\mathrm{q}_{\mathrm{m}}$ - vazão média dos gotejadores $\left(\mathrm{L} \mathrm{h}^{-1}\right)$;

n - número de gotejadores.

O desempenho dos sistemas de irrigação localizada foi classificado segundo o Coeficiente de Uniformidade de Christiansen (CUC), o Coeficiente de Uniformidade de Distribuição (CUD) e o Coeficiente de Uniformidade Estatística (Us) segundo classificação de Mantovani (2001) (Tabela 2).

Tabela 2: Classificação dos valores do desempenho de sistemas de irrigação localizada em função dos coeficientes de Uniformidade de Christiansen (CUC), de Uniformidade de Distribuição (CUD) e de Uniformidade Estatística (Us)

\begin{tabular}{lccc}
\hline Classe & CUC & CUD & Us \\
\cline { 2 - 4 } & \multicolumn{3}{c}{$(\%)$} \\
\hline Excelente & $>90$ & $>84$ & $\geq 90$ \\
Bom & $80-90$ & $68-84$ & $80 \mid-90$ \\
Razoável & $70-80$ & $52-68$ & $70 \mid-80$ \\
Ruim & $60-70$ & $36-52$ & $60 \mid-70$ \\
Inaceitável & $<60$ & $<36$ & $<60$ \\
\hline Fonte: Mantovani (2001).
\end{tabular}

Dantas Neto et al. (2013) relacionam a uniformidade de distribuição de água com a variação de pressão máxima ocorrida nas linhas laterais, e propuseram a Equação 3 para avaliar a variação de vazão ao longo das linhas laterais. Este dado indica quais linhas laterais avaliadas apresentam maiores problemas de funcionamento. 


$$
\Delta \mathrm{Q}=\frac{\left(\mathrm{Q}_{\text {máx }}-\mathrm{Q}_{\text {mín }}\right)}{\mathrm{Q}_{\text {máx }}}
$$

Em que:

$\Delta \mathrm{Q}$ - variação de vazão na linha lateral (\%); Qmáx - valor máximo de vazão na linha lateral $\left(\mathrm{L} \mathrm{h}^{-1}\right)$;

Qmín - valor mínimo de vazão na linha lateral $\left(\mathrm{L} \mathrm{h}^{-1}\right)$.

A caracterização de eficiência agronômica dos sistemas consistiu na avaliação de quanto de água a planta necessita e quanto ela recebe pela aplicação via sistema de irrigação. Silva et al. (2004) afirmam que na obtenção da área adequadamente irrigada, busca-se o ponto ótimo entre percentual de área que deve receber aplicações de água em quantidades iguais ou superiores à lâmina requerida pela cultura para satisfazer suas exigências para pleno desenvolvimento, com menor percentual de excesso possível.

A coleta de amostras de solo para avaliação do manejo da irrigação adotado consistiu na retirada de três amostras de solo, imediatamente antes da irrigação, conforme o manejo adotado pelo produtor, a $15 \mathrm{~cm}$ do emissor que aplica água naquela planta, representando as profundidades de 0-25 e 25-50 cm do perfil do solo, na primeira planta, uma planta localizada no centro da linha e a última planta da linha lateral, em cada linha lateral em que a vazão foi avaliada.

As amostras simples formaram uma amostra composta dentro de cada setor avaliado, que permaneceu acondicionada em recipiente vedado para determinação de umidade atual do solo, seguindo o método gravimétrico (BERNARDO et al., 2019). Parte de cada amostra composta foi utilizada também para determinação da curva de retenção de água.

As determinações de umidade atual e densidade do solo foram realizadas no Laboratório de Solo do Instituto Federal do Espírito Santo (IFES) - Campus Santa Teresa. A análise de retenção de água no solo foi realizada no Laboratório de
Hidráulica, Irrigação e Drenagem do Centro de Ciências Agrárias da Universidade Federal do Espírito Santo (UFES), em Alegre - ES.

De posse dos resultados das análises físicas e hídricas do solo, procederam-se aos cálculos da Irrigação Real Necessária (IRN) para elevar a umidade atual do solo até a Capacidade de Campo, utilizando a Equação 4, proposta por Mantovani et al. (2009).

$$
\Delta Q=\frac{\left(Q_{\text {máx }}-Q_{\text {mín }}\right)}{Q_{\text {máx }}}
$$

Em que:

$\mathrm{IRN}_{\text {loc }}$ - irrigação real necessária, em sistemas de irrigação localizada (mm);

CC - capacidade de campo (\% em peso);

Ua - umidade atual do solo (\% em peso);

Ds - densidade do solo $\left(\mathrm{g} \mathrm{cm}^{-3}\right)$;

$\mathrm{Z}$ - profundidade efetiva do sistema radicular $(\mathrm{cm})$;

PAM - percentagem de área molhada.

As lâminas aplicadas em cada ponto foram ordenadas de forma crescente e correlacionadas com suas frações de áreas irrigadas. Foram então gerados gráficos, que proporcionaram a identificação, dentro de cada setor, da área adequadamente irrigada (\%), isto é, a área que recebeu lâmina igual ou superior à necessidade da cultura.

A lâmina média coletada (mm) é a média aritmética das lâminas coletadas no teste em campo. A lâmina armazenada na zona radicular $(\mathrm{mm})$ identificada através da Equação 5, se refere à lâmina que fica retida na zona radicular.

$$
\mathrm{L}_{\mathrm{arm}}=\mathrm{IRN}-\mathrm{L}_{\text {déf }}
$$

Em que:

Larm - lâmina armazenada na zona radicular (mm);

IRN - irrigação real necessária (mm);

Ldéf - lâmina deficitária (mm).

A lâmina deficitária (mm) corresponde à diferença entre lâmina 
armazenada na zona radicular e a IRN (Equação 6), expressando o quanto de água faltou para atendimento às necessidades da cultura.

$$
\mathrm{L}_{\mathrm{déf}}=\frac{\sum_{i=1}^{n}\left(L_{i}-I R N\right)}{\mathrm{n}}
$$

Em que:

Ldéf - lâmina deficitária (mm), quando (Li IRN) $<0$;

$\mathrm{L}_{\mathrm{i}}$ - lâmina obtida no coletor "i "” (mm); IRN - irrigação real necessária (mm); $\mathrm{n}$ - número de coletores em déficit.

A lâmina percolada (mm) calculada pela Equação 7 é baseada na média do excesso de lâmina aplicada em relação à IRN.

$$
\mathrm{L}_{\mathrm{per}}=\frac{\sum_{i=1}^{n}\left(L_{i}-I R N\right)}{\mathrm{n}}
$$

Em que:

Lper - lâmina percolada (mm), quando ( $\mathrm{Li} \mathrm{-}$ IRN) $>0$;

$\mathrm{L}_{\mathrm{i}}$ - lâmina obtida no coletor “i” (mm);

IRN - irrigação real necessária (mm);

$\mathrm{n}$ - número de coletores em excesso.

Com a relação entre lâmina armazenada na zona radicular e a lâmina aplicada, calculou-se a eficiência de aplicação de água, pela Equação 8.

$$
\mathrm{Ea}=100\left(\frac{L_{\text {arm }}}{\mathrm{L}_{\mathrm{apl}}}\right)
$$

Em que:

Ea - eficiência de aplicação de água (\%);

Larm - lâmina armazenada na zona radicular (mm);

Lapl - lâmina aplicada (mm).

O coeficiente de déficit (\%) é expresso através da razão entre a lâmina d'água deficitária e a lâmina de irrigação real necessária (HART et al., 1979), conforme Equação 9.

$$
\mathrm{Cd}=100\left(\frac{L_{\text {dé } f}}{\mathrm{IRN}}\right)
$$

Em que:

Cd - coeficiente de déficit (\%);

Ldéf - lâmina em déficit (mm);

IRN - irrigação real necessária (mm).

A perda por percolação (\%), é o volume de água aplicada que, através de movimento descendente no perfil do solo, localiza-se abaixo da zona de atuação das raízes, sendo a razão entre a lâmina percolada e a lâmina coletada (Equação 10).

$$
P_{p}=100\left(\frac{L_{p e r}}{\mathrm{~L}_{\mathrm{col}}}\right)
$$

Em que:

$\mathrm{P}_{\mathrm{p}}$ - perdas por percolação (\%);

$\mathrm{L}_{\text {per }}$ - lâmina percolada (mm);

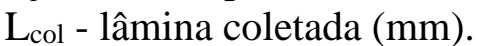

\section{RESULTADOS E DISCUSSÃO}

Os valores referentes aos parâmetros físico-hídricos dos solos nos sistemas avaliados são apresentados na Tabela 3.

Pode-se observar que os valores da capacidade de campo (CC) na tensão de 10 $\mathrm{kPa}$ estão compreendidos entre 16,23\% e $21,87 \%$ na camada de 0 a $25 \mathrm{~cm}$ e entre $18,64 \%$ e $26,64 \%$ na profundidade de 25 a $50 \mathrm{~cm}$. Para os valores de ponto de murcha permanente (PMP), na tensão de $1.500 \mathrm{kPa}$, estes estão compreendidos entre 8,78\% e $17,12 \%$, na profundidade de 0 a $25 \mathrm{~cm}$, $10,54 \%$ e $19,98 \%$ na profundidade de 25 a $50 \mathrm{~cm}$. Os valores de densidade do solo variam de 1,06 a $1,21 \mathrm{~g} \mathrm{~cm}^{-3}$. Os valores de disponibilidade total de água (DTA) variam entre 0,42 a $1,06 \mathrm{~mm} \mathrm{~cm}^{-1}$. 
Tabela 3: Valores de umidade do solo na capacidade de campo (CC), umidade no ponto de murcha permanente (PMP), umidade atual da água do solo (Ua), densidade do solo (Ds) e disponibilidade total de água (DTA) nos sistemas avaliados

\begin{tabular}{|c|c|c|c|c|c|c|}
\hline Sistema & $\begin{array}{c}\text { Camada } \\
(\mathrm{cm})\end{array}$ & $\begin{array}{l}\text { CC } \\
---- \\
\end{array}$ & $\begin{array}{l}\text { PMP } \\
\% \text { peso }\end{array}$ & Ua & $\begin{array}{c}\text { Ds } \\
\left(\mathrm{g} \mathrm{cm}^{-3}\right)\end{array}$ & $\begin{array}{c}\text { DTA } \\
\left(\mathrm{mm} \mathrm{cm}^{-1}\right)\end{array}$ \\
\hline \multirow{2}{*}{1} & $0-25$ & 21,83 & 17,12 & 19,65 & 1,12 & 0,53 \\
\hline & $25-50$ & 23,91 & 19,98 & 21,57 & 1,07 & 0,42 \\
\hline \multirow{2}{*}{2} & $0-25$ & 18,76 & 11,63 & 16,82 & 1,08 & 0,77 \\
\hline & $25-50$ & 21,81 & 12,23 & 19,20 & 1,06 & 1,02 \\
\hline \multirow{2}{*}{3} & $0-25$ & 16,87 & 10,76 & 15,08 & 1,09 & 0,67 \\
\hline & $25-50$ & 19,26 & 12,03 & 16,61 & 1,08 & 0,78 \\
\hline \multirow{2}{*}{4} & $0-25$ & 16,23 & 10,87 & 13,36 & 1,12 & 0,60 \\
\hline & $25-50$ & 19,54 & 12,78 & 19,78 & 1,09 & 0,74 \\
\hline \multirow{2}{*}{5} & $0-25$ & 21,87 & 14,98 & 22,01 & 1,16 & 0,80 \\
\hline & $25-50$ & 26,64 & 17,10 & 28,33 & 1,11 & 1,06 \\
\hline \multirow[b]{2}{*}{6} & $0-25$ & 16,98 & 8,78 & 12,74 & 1,21 & 0,99 \\
\hline & $25-50$ & 18,64 & 10,54 & 16,18 & 1,13 & 0,92 \\
\hline \multirow{2}{*}{7} & $0-25$ & 19,22 & 12,75 & 15,67 & 1,07 & 0,69 \\
\hline & $25-50$ & 23,46 & 15,32 & 19,76 & 1,07 & 0,87 \\
\hline \multirow{2}{*}{8} & $0-25$ & 18,55 & 12,91 & 14,32 & 1,12 & 0,63 \\
\hline & $25-50$ & 22,76 & 15,72 & 16,29 & 1,08 & 0,76 \\
\hline \multirow{2}{*}{9} & $0-25$ & 19,49 & 11,27 & 10,93 & 1,13 & 0,93 \\
\hline & $25-50$ & 22,43 & 16,89 & 16,97 & 1,09 & 0,60 \\
\hline \multirow{2}{*}{10} & $0-25$ & 17,32 & 10,62 & 15,91 & 1,14 & 0,76 \\
\hline & $25-50$ & 20,08 & 13,03 & 18,99 & 1,06 & 0,75 \\
\hline
\end{tabular}

Pode-se observar que os valores da capacidade de campo (CC) na tensão de 10 $\mathrm{kPa}$ estão compreendidos entre 16,23\% e $21,87 \%$ na camada de 0 a $25 \mathrm{~cm}$ e entre $18,64 \%$ e $26,64 \%$ na profundidade de 25 a $50 \mathrm{~cm}$. Para os valores de ponto de murcha permanente (PMP), na tensão de $1.500 \mathrm{kPa}$, estes estão compreendidos entre 8,78\% e $17,12 \%$, na profundidade de 0 a $25 \mathrm{~cm}$, $10,54 \%$ e $19,98 \%$ na profundidade de 25 a $50 \mathrm{~cm}$. Os valores de densidade do solo variam de 1,06 a $1,21 \mathrm{~g} \mathrm{~cm}^{-3}$. Os valores de disponibilidade total de água (DTA) variam entre 0,42 a $1,06 \mathrm{~mm} \mathrm{~cm}^{-1}$.

Os valores médios da CC são de $18,71 \%$ na profundidade de 0 a $25 \mathrm{~cm}$ e $21,85 \%$ na profundidade de 25 a 50 m. Já os valores médios do PMP são de 12,17\% na profundidade de 0 a $25 \mathrm{~cm}$, e $14,56 \%$ na profundidade de 25 a $50 \mathrm{~cm}$.

Observa-se que o solo do sistema 5 apresenta os maiores valores de CC (21,87\% e 26,64\%), e para o PMP, o sistema 1 apresenta os maiores valores $(17,12 \%$ e $19,98 \%)$ para ambas as profundidades. O menor valor de CC na profundidade 0 a $25 \mathrm{~cm}$ foi encontrado no sistema 3 (16,87\%), e na profundidade de 25 a $50 \mathrm{~cm}$, o menor valor foi encontrado no sistema 6 (18,64\%). Os menores valores de PMP foram encontrados no solo do sistema $6(8,78 \%$ e $10,54 \%)$.

Quanto à densidade, o solo do sistema 6 apresenta os maiores valores nas duas profundidades $\left(1,21\right.$ e $\left.1,13 \mathrm{~g} \mathrm{~cm}^{-3}\right)$, e os menores valores estão no solo do sistema $7\left(1,07 \mathrm{~g} \mathrm{~cm}^{-3}\right)$ na profundidade de 0 a 25 $\mathrm{cm}$, e nos sistemas 2 e $10\left(1,06 \mathrm{~g} \mathrm{~cm}^{-3}\right)$ na profundidade de 25 a $50 \mathrm{~cm}$.

Observa-se através da Tabela 4, que no sistema 5, a irrigação foi realizada no momento em que a umidade do solo estava superior à capacidade de campo, indicando que a lâmina em sua totalidade foi perdida por percolação profunda. Em $70 \%$ dos sistemas avaliados (1, 2, 3, 4, 6, 7 e 10), as irrigações foram realizadas enquanto o solo 
estava dentro dos limites aceitáveis de déficit, respeitando-se o fator de segurança. Ainda, 20\% dos sistemas (8 e 9), receberam irrigações com déficit de água no solo acima do limite recomendado de disponibilidade de água no solo para a cultura do café, que é de $50 \%$.

Tabela 4: Resultados dos parâmetros de avaliação do manejo de irrigação para os projetos de irrigação localizada: profundidade efetiva do sistema radicular (Z), porcentagem de área molhada (Pam), capacidade total de armazenamento de água (CTA), capacidade real de armazenamento de água (CRA), irrigação real necessária $\left(\mathrm{IRN}_{\mathrm{LOC}}\right)$ e déficit de água no solo (Def)

\begin{tabular}{lcccccc}
\hline Sistema & $\begin{array}{c}\text { Z } \\
(\mathrm{cm})\end{array}$ & $\begin{array}{c}\text { PAM } \\
(\%)\end{array}$ & $\begin{array}{c}\text { CTA } \\
(\mathrm{mm})\end{array}$ & $\begin{array}{c}\text { CRA } \\
(\mathrm{mm})\end{array}$ & $\begin{array}{c}\mathrm{IRN}_{\text {Loc }} \\
(\mathrm{mm})\end{array}$ & $\begin{array}{c}\text { Def } \\
(\%)\end{array}$ \\
\hline 1 & 50 & 23,2 & 5,5 & 2,7 & 2,8 & 51,5 \\
2 & 50 & 23,8 & 10,6 & 5,3 & 2,5 & 23,5 \\
3 & 50 & 32,1 & 11,6 & 5,8 & 3,1 & 27,0 \\
4 & 50 & 18,4 & 6,2 & 3,1 & 3,0 & 48,1 \\
5 & 50 & 21,5 & 10,0 & 5,0 & 0,0 & 0,0 \\
6 & 50 & 18,0 & 8,6 & 4,3 & 4,6 & 53,8 \\
7 & 50 & 28,3 & 11,1 & 5,5 & 5,4 & 48,6 \\
8 & 50 & 20,1 & 7,0 & 3,5 & 4,8 & 68,1 \\
9 & 50 & 29,6 & 11,3 & 5,7 & 14,3 & 126,2 \\
10 & 50 & 26,7 & 10,1 & 5,0 & 2,1 & 21,3 \\
\hline
\end{tabular}

valor de CUC classificado como razoável,

O sistema 9 apresentou déficit acima de $100 \%$, em que a Ua se encontrava abaixo do PMP. Foi constatado neste local, danos severos pela falta de água à cultura, como sistema radicular superficial com alto índice de mortalidade, redução na produção e no crescimento compensatório.

Os valores de coeficiente de uniformidade de Christiansen (CUC), coeficiente de uniformidade de distribuição (CUD), uniformidade estatística (Us) e suas respectivas classificações e variação de vazão $(\Delta Q)$ são apresentados na Figura 2.

O valor médio do CUC foi de $76,5 \%$, com variação de $69,7 \%$ a $82,4 \%$. Apenas dois dos sistemas avaliados (4 e 6) apresentam CUC acima de $80 \%$, sendo classificados como bom. Um sistema avaliado (10) apresentou CUC abaixo de $70 \%$ sendo classificado como ruim, e os outros sete sistemas avaliados tiveram seu

\section{com valores situados entre $70 \%$ e $80 \%$.}

Ao considerar o CUD, que imprime maior restrição na avaliação, por considerar os $25 \%$ menores valores de vazão em relação à média, verifica-se que 4 sistemas foram classificados como bom (2, 4, 6 e 8) com seus valores compreendidos entre $68 \%$ e $84 \%$, e os demais sistemas foram classificados como razoável, com valores entre $52 \%$ e $68 \%$.

Os baixos valores de uniformidade de aplicação de água observados, sobretudo nos sistemas 3, 5 e 10 são devidos, principalmente, à instalação dos sistemas de irrigação sem dimensionamento de projeto técnico realizado por profissional capacitado, fazendo com que o sistema opere com baixa pressão de serviço, conforme observa-se na Tabela 5. Soma-se ainda, ocorrência de obstruções parciais de emissores nas linhas laterais.

Figura 2: Valores do Coeficiente de Uniformidade de Christiansen (CUC), Coeficiente de Uniformidade de Distribuição (CUD), Uniformidade Estatística (Us), classificação dos coeficientes dos projetos de irrigação localizada de acordo com Mantovani (2001) e variação de vazão $(\Delta \mathrm{Q})$ média nas linhas laterais. 


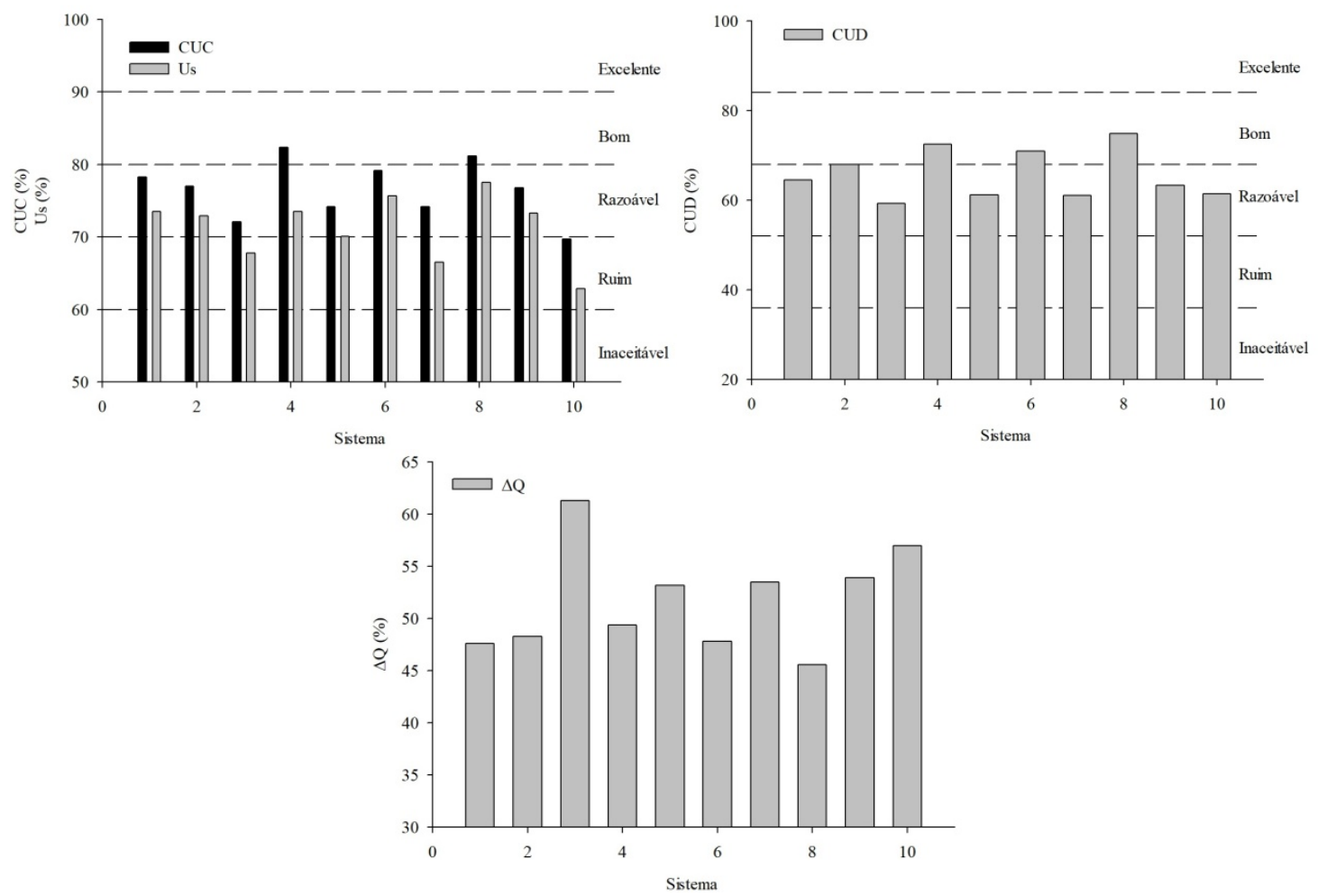

Tabela 5: Dados dos sistemas avaliados, com Fabricante, Pressão nominal (Pn), Pressão medida (Pm), Vazão nominal (Qn), vazão medida (Qm).

\begin{tabular}{|c|c|c|c|c|c|}
\hline \multirow[t]{2}{*}{ Sistema } & \multirow[t]{2}{*}{ Fabricante } & Pn & $\mathrm{Pa}$ & Qn & $\mathrm{Qa}$ \\
\hline & & \multicolumn{2}{|c|}{$\mathrm{kPa}$} & \multicolumn{2}{|c|}{$\mathrm{L} \mathrm{h}^{-1}$} \\
\hline 1 & NaanDanJain & 150 & 145 & 1,7 & 1,7 \\
\hline 2 & Petroisa & 100 & 97 & 1,9 & 1,9 \\
\hline 3 & Petroisa & 100 & 75 & 2,1 & 1,9 \\
\hline 4 & Não identificado & - & 100 & - & 1,7 \\
\hline 5 & Petroisa & 100 & 80 & 2,1 & 1,9 \\
\hline 6 & Azud Line & 100 & 100 & 2,1 & 2,1 \\
\hline 7 & Não identificado & - & 120 & - & 2,0 \\
\hline 8 & Não identificado & - & 120 & - & 1,7 \\
\hline 9 & NaanDanJain & 150 & 150 & 1,7 & 1,7 \\
\hline 10 & Azud Line & 120 & 90 & 2,1 & 1,9 \\
\hline
\end{tabular}

Os sistemas 1, 2, 4 e 8 apresentaram bons índices de uniformidade de aplicação de água, porém abaixo do que é esperado para sistemas de irrigação localizada por gotejamento. Sua instalação ocorreu após prévio dimensionamento, porém, a falta de manutenção comprometeu o funcionamento pleno do sistema, provocando obstrução parcial de alguns emissores.

Estes valores ficaram bem abaixo do que foi obtido por Martins et al. (2013), que avaliou sistemas de irrigação localizada em café Conilon no sul do Espírito Santo, e a do tipo gotejamento apresentou CUC de 92,39\% e CUD de $90,54 \%$, sendo, portanto, classificados como "excelente".

Estes valores são também inferiores aos de Gottardo (2016), que avaliou um sistema de irrigação localizada por gotejamento em Campos dos Goytacazes, RJ, apresentando valores de CUC de 93,13\% e CUD de 88,5\%, classificados como "excelente". 
Sete dos sistemas avaliados apresentam Us classificados como razoável, e os demais são classificados como ruim. Bonomo (1999) classificou dois sistemas como "razoável" e outros dois como "ruim", ao avaliar irrigação localizada por gotejamento em regiões cafeeiras do Triângulo e Noroeste de Minas Gerais. Santos et al. (2012) obtiveram resultados distintos ao avaliarem três lotes de irrigação em Jaguaribe - CE, onde classificaram os lotes como “razoável” (71\%), “bom” (81\%) e “excelente” (90\%).

A $\Delta \mathrm{Q}$ de todos os sistemas avaliados encontra-se muito acima do obtido por Dantas Neto (2013), que obteve médias de $15,8 \%$ e $22,0 \%$ em dois sistemas de irrigação localizada por gotejamento em subárea do Perímetro Irrigado de Sumé. Caitano et al. (2011) também obtiveram valores inferiores aos obtidos neste estudo, ao avaliarem sistemas de irrigação localizada por microaspersão e gotejamento no Perímetro Irrigado Baixo Acaraú. Os valores encontrados nas três avaliações são elevados, acima do recomendado por Keller e Karmeli (1975), que é de $10 \%$ para emissores nas extremidades, funcionando simultaneamente.

O sistema 4, que apresentou maior valor de CUC, possui maior variação de vazão nos últimos emissores avaliados, evidenciando que seu desempenho é comprometido pela ausência de práticas de limpeza no sistema. Enquanto o sistema 10, de menor CUC, possui 3 linhas laterais com altos valores de vazão no início, que apresentam decréscimo acentuado no decorrer destas linhas, que é causado também pela ausência de limpeza do sistema, mas também com a baixa pressão em que o sistema opera.

A uniformidade de todos os sistemas pode ser melhorada através de práticas simples de manutenção preventiva e corretiva, como simples limpeza do sistema de filtragem e dos emissores obstruídos, ou até mesmo sua substituição.

Na Tabela 6 são apresentados os valores de Irrigação Real Necessária (IRN $\left.\mathrm{Loc}_{\mathrm{L}}\right)$, lâmina aplicada $\left(\mathrm{L}_{\mathrm{apl}}\right)$, lâmina armazenada ( $\left.\mathrm{L}_{\mathrm{arm}}\right)$, lâmina deficitária ( $\left.\mathrm{L}_{\mathrm{def}}\right)$ e lâmina percolada $\left(\mathrm{L}_{\mathrm{per}}\right)$ dos sistemas avaliados.

Os valores de IRN $\mathrm{N}_{\text {Loc }}$ variam de 0 a 14,3 mm nos sistemas avaliados. O sistema 5 apresentou $\mathrm{IRN}_{\text {Loc }}$ de $0 \mathrm{~mm}$, devido sua Ua estar acima da CC, onde no momento, não era necessário que fosse aplicada qualquer lâmina d'água. O manejo de irrigação não é realizado com qualquer critério técnico, sendo adotado turno de rega fixo.

Tabela 6: Irrigação real necessária (IRN $\mathrm{Loc}$ ), lâmina aplicada (Lapl), lâmina armazenada na zona radicular ( $\mathrm{L}_{\mathrm{arm}}$ ), lâmina deficitária ( $\mathrm{L}_{\mathrm{def}}$ ) e lâmina percolada $\left(\mathrm{L}_{\mathrm{per}}\right)$

\begin{tabular}{llllll}
\hline \multirow{2}{*}{ Sistema } & IRN $_{\text {Loc }}$ & $\mathrm{L}_{\text {apl }}$ & $\mathrm{L}_{\text {arm }}$ & $\mathrm{L}_{\text {def }}$ & $\mathrm{L}_{\text {per }}$ \\
& --------------- & $\mathrm{mm}$ & ------------ \\
\hline 1 & 2,8 & 3,6 & 2,8 & 0,0 & 0,8 \\
2 & 2,5 & 3,7 & 2,5 & 0,0 & 1,2 \\
3 & 3,1 & 4,2 & 3,1 & 0,0 & 1,1 \\
4 & 3,0 & 5,1 & 3,0 & 0,0 & 2,1 \\
5 & 0,0 & 4,2 & 0,0 & 0,0 & 4,2 \\
6 & 4,6 & 4,6 & 4,6 & 0,0 & 0,0 \\
7 & 5,4 & 4,3 & 4,3 & 1,1 & 0,0 \\
8 & 4,8 & 3,7 & 3,7 & 1,1 & 0,0 \\
9 & 14,3 & 3,7 & 3,7 & 10, & 0,0 \\
10 & 2,1 & 4,2 & 2,1 & 0,0 & 2,1 \\
\hline
\end{tabular}

Pode-se atentar que, em sete dos sistemas, a água foi aplicada em excesso, isto é, $\mathrm{L}_{\mathrm{apl}}$ acima da $\mathrm{IRN}_{\mathrm{Loc}}$, indicando que houve perda por percolação. Além de água, ocorre desperdício de energia elétrica e lixiviação de nutrientes para camadas de solo mais profundas, onde o sistema radicular não explora.

Outros três sistemas aplicaram água em quantidade menor que a necessidade momentânea das plantas, onde o maior déficit foi observado no sistema 9. Isso ocorreu devido à insuficiente 
disponibilidade de água existente na propriedade.

Como já era esperado, o maior valor de $L_{\text {per }}$ foi referente ao sistema 5 , onde a irrigação foi realizada no momento em que a umidade atual do solo estava mais alta que a CC do solo. Na Tabela 7, a Pad apresenta a área adequadamente irrigada dos sistemas avaliados, que representa área que recebeu lâmina d’água igual ou superior ao necessário para suprir as necessidades da planta, com o menor desperdício possível. Observa-se que o sistema 6 possui maior percentual de área adequadamente irrigada, de 62,0\%, enquanto o sistema 7 apresentou o menor valor, de $22 \%$.

Tabela 7: Porcentagem de área adequadamente irrigada (Pad); coeficiente de déficit (Cd); perdas por percolação (Pper); e eficiência de aplicação (Ea)

\begin{tabular}{|c|c|c|c|c|}
\hline Sistema & Pad & $\mathrm{Cd}$ & Pper & $\mathrm{Ea}$ \\
\hline 1 & 59,6 & 0,0 & 22,2 & 77,8 \\
\hline 2 & 42,0 & 0,0 & 32,4 & 67,6 \\
\hline 3 & 40,0 & 0,0 & 26,2 & 73,8 \\
\hline 4 & 51,3 & 0,0 & 41,2 & 58,8 \\
\hline 5 & 41,0 & 0,0 & 100,0 & 0,0 \\
\hline 6 & 62,0 & 0,0 & 0 & 100,0 \\
\hline 7 & 22,0 & 20,5 & 0 & 100,0 \\
\hline 8 & 49,1 & 23,1 & 0 & 100,0 \\
\hline 9 & 49,2 & 74,0 & 0 & 100,0 \\
\hline 10 & 40,0 & 0,0 & 50,0 & 50,0 \\
\hline
\end{tabular}

O Cd é maior para o sistema 9 (74,0\%), pois o mesmo teve $\mathrm{L}_{\text {apl }}$ muito inferior à $\mathrm{IRN}_{\mathrm{Loc}}$, seguido dos sistemas 8 $(23,1 \%)$ e 7 (20,5\%). Os demais sistemas apresentaram $\mathrm{Cd}$ de $0,0 \%$, visto que o valor de $\mathrm{L}_{\text {apl }}$ foi inferior à IRN $\mathrm{ILc}_{\mathrm{Loc}}$ resultando no armazenamento no perfil do solo de todo volume de água aplicado pelo sistema de irrigação. $O$ sistema 6 apresentou Cd e $\mathrm{P}_{\text {per }}$ de $0,0 \%$, visto que sua $\mathrm{L}_{\text {apl }}$ foi igual à IRN $\mathrm{N}_{\text {Loc. }} \mathrm{O}$ sistema 5 obteve $\mathrm{P}_{\text {per }}$ de $100 \%$, sendo o maior valor, causado pela irrigação no momento em que a Ua se encontrava mais alta que a CC. Em seguida, vieram os sistemas 10 (50,0\%), 4
(41,2\%), 2 (32,4\%), 3 (26,2\%) e 1 $(22,2 \%)$.

Ao considerar a Ea, os sistemas 6, 7, 8 e 9 apresentaram valor de $100 \%$, fator relacionado ao armazenamento no perfil do solo de toda a água aplicada pelo sistema de irrigação. Em contrapartida, o sistema 5 apresentou $0,0 \%$ de eficiência, pois toda a água aplicada foi perdida por percolação profunda.

A ausência de projeto técnico elaborado por profissional capacitado aliado à ausência de manutenção e ao manejo inadequado da irrigação induz o desperdício de água, fertilizantes do solo nos sistemas localizados, que geralmente são diretamente associados à economia de água. Tal fator torna essencial que a utilização de água na irrigação seja realizada de maneira mais eficiente, o que irá acarretar, diretamente, maior produtividade da cultura e conservação do meio ambiente, que vem se apresentando como grande desafio da agricultura irrigada.

A irrigação localizada por gotejamento possui maior potencial para uso eficiente da água, mas devido à sua maior sensibilidade de entupimento dos emissores, necessidade de manutenção com maior frequência e também ausência de manejo adequado, deve-se analisar sobre a decisão por sua utilização ou outro método menos sensível, como a aspersão convencional.

\section{CONCLUSÕES}

Todos os sistemas de irrigação localizada por gotejamento apresentaram valores de CUC, CUD e Us inferiores aos recomendados; $\mathrm{Em} \quad 60 \%$ dos projetos avaliados, a lâmina aplicada foi superior à irrigação real necessária à cultura; Em 20\% dos sistemas avaliados, as irrigações foram iniciadas com déficit de água no solo acima do limite recomendado (50\% de disponibilidade); Em 20\% dos sistemas 
avaliados, a perda por percolação foi igual ou superior a $50 \%$.

\section{REFERÊNCIAS}

BERNARDO, S.; MANTOVANI, E. C.; SILVA, D. D; SOARES, A. A. Manual de Irrigação. 9. Ed. Viçosa: UFV 2019. 545 p.

BONOMO, R. Análise da irrigação na cafeicultura em áreas de cerrado de Minas Gerais. 1999. 224 f. Tese (Doutorado em Engenharia Agrícola). Universidade Federal de Viçosa, Viçosa. 1999.

CAITANO, R. F.; LOPES, F. B.; SOUZA, F.; MENDONÇA, M. A. B. Desempenho dos sistemas de irrigação na cultura da banana no Perímetro Irrigado Baixo Acaraú, Ceará. Revista Brasileira de Agricultura Irrigada, v.5, n.2, p.113-122, 2011.

CARVALHO, C. M.; ELOI, W. M.; LIMA, S. C. R. V.; PEREIRA, J. M. G. Desempenho de um sistema de irrigação por gotejamento na cultura da goiaba. Irriga, v.11, n.1, p.36-46, 2006.

CETCAF - CENTRO DE DESENVOLVIMENTO TECNOLÓGICO DO CAFÉ. Cafeicultura Capixaba.

Disponível em < https://www.cetcaf.com.br/cafeiculturacapixaba-e-estatistica>. Acesso em: 16 setembro 2020.

DANTAS NETO, J.; AZEVEDO, C. A. V.; SILVA, L. F. D.; SILVA, P. F.;

SANTOS, C. S. Desempenho de sistema de irrigação por gotejamento em áreas de pequenos produtores do semiárido paraibano. Enciclopédia Biosfera, v.9, n.16, p.679-688, 2013.

DARDENGO, M. C. J. D. Crescimento, produtividade e consumo de água do cafeeiro Conilon sob manejo irrigado e de sequeiro. 2012. $97 \mathrm{f}$. Tese (Doutorado em Produção Vegetal). Universidade Estadual do Norte Fluminense Darcy Ribeiro, Campos dos Goytacazes. 2012.

DENÍCULI, W.; BERNARDO, S.; THIÁBAUT, J. T. L.; SEDYAMA, G. C. Uniformidade de distribuição de água, em condições de campo, num sistema de irrigação por gotejamento. Revista Ceres, v. 27, n. 50, p. 155-162, 1980.

FARIA, L. F.; COELHO, R. D.; RESENDE, R. S. Variação de vazão de gotejadores de fluxo normal enterrados na irrigação de café. Engenharia Agrícola, v. 24, n. 3, p. 589-602, set./dez. 2004.

GOTTARDO, R. D. Desenvolvimento inicial de Coffea canephora submetidos à irrigação superficial e subsuperficial em Campos dos Goytacazes - RJ. 2016. $76 f$. Dissertação (Mestrado em Produção Vegetal). Universidade Estadual do Norte Fluminense Darcy Ribeiro, Campos dos Goytacazes, 2016.

HART, W. E.; PERI, G.; SKOGERBOE, G. V. Irrigation performance: evaluation. Journal of the Irrigation and Drainage Division, v. 105, n. R3, p. 275-288, 1979.

IBGE - Instituto Brasileiro de Geografia e Estatística. Disponível em $<$ http://downloads.ibge.gov.br/downloads_ geociencias.htm>. 2014. Acesso em: 15 abr. 2019.

KELLER, J., KARMELI, D. Trickle irrigation design. Glendora: Rain Bird Sprinkler Manufacturing, 1975. 133 p

MANTOVANI, E. C. Avalia: Programa de Avaliação da Irrigação por Aspersão e Localizada. Viçosa: UFV, 2001.

MANTOVANI, E. C.; BERNARDO, S.; PALARETTI, L. F. Irrigação: princípios e métodos. $3^{\mathrm{a}}$ ed., atual. $3^{\mathrm{a}}$ reimpressão. Viçosa: Ed. UFV, 2009. 
MARTINS, C. L.; BUSATO, C.; SILVA, S. F.; RODRIGURES, W. N.; REIS, E. F. Avaliação do desempenho de sistemas de irrigação no sul do Estado do Espírito Santo.Agro@mbiente Online, v. 7, n. 2, p. 236-241, 2013.

RODRIGUES, R. R.; COLA, M. P. A.; NAZÁRIO, A. A.; AZEVEDO, J. M. G.; REIS, E. F. Eficiência e uniformidade de um sistema de irrigação por gotejamento na cultura do cafeeiro. Ambiência, v. 9, n. 2, p. 323-334, 2013.

SANTOS, D. A.; FREIRE, F. G. C.; SANTOS, F. S. S.; SANTOS, M. M. S.; LIMA, R. M. S.; SANTOS, W. O.

Avaliação da uniformidade de aplicação de água na irrigação por microaspersão na cultura da banana em São João do Jaguaribe - CE. Inovagri, Fortaleza, p.14, 2012.

SILVA, E. M.; LIMA, J. E. F. W.; AZEVEDO, J. A.; RODRIGUES, L. N. Proposição de um modelo matemático para avaliação de desempenho de sistemas de irrigação. Pesquisa Agropecuária

Brasileira, v. 39, n. 8, p. 741-748, 2004.

VIEIRA, G. H. S.; MANTOVANI, E. C.; CORDEIRO, E. A. Avaliação de sistemas de irrigação por gotejamento: comparação das metodologias propostas por Keller e por Denículi. Em: VI Simpósio de Pesquisa em Cafeicultura Irrigada FENICAFÉ, 2003, Araguari. Anais. Brasília: Embrapa Café, 2003.

VIEIRA, G. H. S.; MANTOVANI, E. C.; SILVA, J G. F.; RAMOS, M. M.; SILVA, C. M. Recuperação de gotejadores obstruídos devido à utilização de águas ferruginosas. Revista Brasileira de Engenharia Agrícola e Ambiental, v. 8, n. 1, p.1-6. 2004.

\section{ZOCOLER, J. L. Avaliação de} desempenho de sistemas de irrigação.

Departamento de Fitossanidade, Engenharia Rural e Solos - Área de Hidráulica e Irrigação. Faculdade de Engenharia de Ilha Solteira - UNESP. Disponível em $<$ http://www.agr.feis.unesp.br/curso5.htm> . Acesso em: 26 mar. 2015. 